\title{
Glossing abbreviations
}

The system of interlinear glossing as practiced in this volume is based on the Leipzig Glossing Rules developed by members of the Department of Linguistics of the Max Planck Institute for Evolutionary Anthropology and the Department of Linguistics of the University of Leipzig.

\section{Punctuation}

Parallel in the object-language/transliteration and the meta-language/gloss:

- Connects segmentable morphemes.

$=$ Marks clitic boundaries.

Indicates reduplication morphemes.

Isolated in the object-language/transliteration or the meta-language/gloss:

- Separates two meta-language elements that correspond to a single object-language element (that cannot be separated into corresponding different morphemes).

: Separates two meta-language elements that correspond to a single object-language element that could be separated into corresponding different morphemes, but the author chose not to separate it; or it separates two object-language elements that correspond to a single meta-language element that could be separated into corresponding different morphemes, but the author chose not to separate it.

- Combines two meta-language elements that correspond to a single object-language element; or it combines two unconnected object-language elements that correspond to a single meta-language element.

I Marks a grammatical property in the object-language signaled by a morpho-phonological change (ablaut, mutation, tone alternation, etc.)

Different meanings in the meta-language/gloss and the object-language transliteration:

... in the meta-language:

( ) Marks inherent, non-overt categories.

[ ] Marks a property that does not correspond to an overt element in the object-language.

... in the object-language/philological transliteration:

() Marks scholarly reconstruction of non-overt phonemes in writing systems with 'defective' spellings, e.g. unwritten glides in Egyptian.

「 1 Marks a passage in the written object-language that is partially destroyed.

[ ] Marks a passage in the written object-language that is completely destroyed; eventually it contains scholarly reconstructions of the destroyed passage.

\{ \} Marks extra elements in the object-language that are to be classified either as scribal errors (philological emendation) or as redundant information as part of certain orthographical conventions .

〈〉 Marks additions to the object-language data, whose missing is to be classified as scribal errors (philological emendation).

* Marks reconstructed forms. 


\section{Abbreviations}

\begin{tabular}{|c|c|}
\hline 1 & first person \\
\hline 2 & second person \\
\hline 3 & third person \\
\hline ABL & ablative \\
\hline ABSTR & abstractum \\
\hline ACC & accusative \\
\hline $\mathrm{ACT}$ & active \\
\hline ADJ & adjective \\
\hline ADJZ & adjectivizer/adjectivization \\
\hline $\mathrm{ADV}$ & adverb(ial) \\
\hline ADVZ & adverbializer/adverbialization \\
\hline AGR & agreement \\
\hline AGT & agent marker \\
\hline ALL & allative \\
\hline ANT & anterior \\
\hline AOR & aorist \\
\hline APPL & applicative \\
\hline ART & article \\
\hline ATTD & attached \\
\hline ATTN & attention catching particle \\
\hline BASE & $\begin{array}{l}\text { particle as base for enclitic } \\
\text { pronoun }\end{array}$ \\
\hline BEN & benefactive \\
\hline $\mathrm{C}$ & communis \\
\hline CAUS & causative \\
\hline CIRC & circumferential \\
\hline CJVB & conjunctional verb \\
\hline $\mathrm{CL}$ & $\begin{array}{l}\text { (written) classifier } \\
\text { (trad. 'determinative') }\end{array}$ \\
\hline CNSV & consecutive particle or suffix \\
\hline COLL & collective \\
\hline COND & conditional \\
\hline CONN & connective particle \\
\hline $\mathrm{COP}$ & copula \\
\hline CORD & coordinating particle \\
\hline DAT & dative \\
\hline DATLOC & dative-locative \\
\hline $\mathrm{DEF}$ & definite \\
\hline DEM & demonstrative \\
\hline DIST & distal \\
\hline DISTR & distributive \\
\hline DU & dual \\
\hline ELAT & elative \\
\hline XCL & exclusive \\
\hline
\end{tabular}

\begin{tabular}{|c|c|}
\hline EXCLM & exclamative \\
\hline EXTR & exterior \\
\hline $\mathrm{F}$ & feminine \\
\hline FUT & future \\
\hline GEN & genitive \\
\hline GN & god's name \\
\hline GRND & ground \\
\hline ILL & illative \\
\hline IMP & imperative \\
\hline IMPRS & impersonal \\
\hline INDF & indefinite \\
\hline INESS & inessive \\
\hline INF & infinitive \\
\hline INFR & inferior \\
\hline INS & instrumental \\
\hline IPFV & imperfective \\
\hline IPRF & imperfect \\
\hline LOC & locative \\
\hline LOCADV & locative-adverbialis \\
\hline M & masculine \\
\hline MED & medium \\
\hline MOD & modal \\
\hline MODP & modal particle \\
\hline MP & medio-passive \\
\hline $\mathrm{N}$ & neuter \\
\hline N... & non- (e.g.npst non-past) \\
\hline NEG & negation, negative \\
\hline NINFL & $\begin{array}{l}\text { not inflected (here: for gender } \\
\text { and number) }\end{array}$ \\
\hline NMLZ & nominalizer/nominalization \\
\hline NOM & nominative \\
\hline OBJ & object \\
\hline OBLV & obligative \\
\hline OBP & Ortsbezugspartikel \\
\hline OPT & optative \\
\hline ORD & ordinal number \\
\hline PASS & passive \\
\hline PERS & personal \\
\hline PFV & perfective \\
\hline PL & plural \\
\hline PLUPRF & plu-perfect \\
\hline PN & personal name \\
\hline POSS & possessive \\
\hline PP & adpositional phrase \\
\hline
\end{tabular}




$\begin{array}{llll}\text { PREP } & \text { preposition } & \text { SBRD } & \text { subordinating particle } \\ \text { PRF } & \text { perfect } & \text { SG } & \text { singular } \\ \text { PROH } & \text { prohibitive } & \text { SP } & \text { sentence particle } \\ \text { PROX } & \text { proximal/proximate } & \text { STAT } & \text { stative } \\ \text { PRS } & \text { present } & \text { STC } & \text { status constructus } \\ \text { PRT } & \text { preterite (= PST past) } & \text { STPR } & \text { status pronominalis } \\ \text { PST } & \text { past } & \text { SUPR } & \text { superior } \\ \text { PTCL } & \text { particle } & \text { TA } & \text { tense/aspect gram } \\ \text { PTCP } & \text { participle } & \text { TAM } & \text { tense/aspect/mode gram } \\ \text { QUOT } & \text { quotative } & \text { TERM } & \text { terminative-adverbialis } \\ \text { RECP } & \text { reciprocal } & \text { THMZ } & \text { thematizer/thematization } \\ \text { REFL } & \text { reflexive } & \text { TN } & \text { toponym } \\ \text { REL } & \text { relative } & \text { TOP } & \text { topic } \\ \text { RES } & \text { resultative } & \text { TR } & \text { transitive } \\ \text { SBJN } & \text { subjunction } & \text { VENT } & \text { ventive } \\ \text { SBJV } & \text { subjunctive } & \text { VOC } & \text { vocative }\end{array}$

\section{Bibliography}

\section{Di Biase-Dyson, Kammerzell \& Werning 2009}

Camilla Di Biase-Dyson, Frank Kammerzell \& Daniel A. Werning, Glossing Ancient Egyptian: Suggestions for Adapting the Leipzig Glossing Rules, in: Lingua Aegyptia. Fournal of Egyptian Language Studies 17 (2009), 243-266; < http://www.gwdg.de/ dwernin/published/DiBiase_ Kammerzell_Werning-2009-Glossing_Ancient_Egyptian.pdf>.

\section{Glossing Ancient Languages}

Glossing Ancient Languages, Open access Wiki, http://wikis.hu-berlin.de/interlinear_glossing/, edited by Daniel A. Werning, Berlin: Humboldt University Berlin.

\section{Leipzig Glossing Rules}

The Leipzig Glossing Rules: Conventions for Interlinear Morpheme-by-Morpheme Glosses, ed. by the Department of Linguistics of the Max Planck Institute for Evolutionary Anthropology (Bernard Comrie, Martin Haspelmath) and by the Department of Linguistics of the University of Leipzig (Balthasar Bickel); <http://www.eva.mpg.de/lingua/resources/glossing-rules.php>, $<$ http://www.eva.mpg.de/lingua/pdf/LGR08.02.05.pdf>, Leipzig, Sept., 12th 2008 (download 2009). 
